\title{
A Comparative Study on Image Compression Method Using Stand-Alone DWT Techniques and Hybrid of DWT with Huffman Coding Technique for WSN Application
}

\author{
Mumtaz binti Anwar Hussin*1, Farhana binti Ahmad Poad*2, Ariffuddin bin Joret*3 \\ *Department of Communication Engineering, Faculty of Electrical and Electronic Engineering, \\ Universiti Tun Hussein Onn Malaysia, 86400 Parit Raja, Batu Pahat, Johor, Malaysia. \\ ${ }^{1}$ ge180091@ siswa.uthm.edu.my \\ ${ }^{2}$ farhana@uthm.edu.my \\ 3ariff@uthm.edu.my
}

\begin{abstract}
The advent of wireless technologies nowadays gives high impact toward many users to communicate to each other. Wireless Sensor Net-work is a network of nodes which connect to each other by using a device. In addition, transmitting and receiving messages and files are common in most users. There are many platform of network which contributes the same aim that is communication purposes. A ZigBee network is known as one of a platform with its own Standard (IEEE 802.15.4). Unfortunately, ZigBee has a low data rate which limits the capacity of storage in transmitting data. Thus, a large multimedia such as image data are hard to transmit via ZigBee network. Therefore, image compression are necessary in transmission process due to the ability in reducing dimension size and removing redundant image data. In this paper, three method are observed which are stand-alone DWT, stand-alone Huffman, and Hybrid of DWT and Huffman Coding. After conducting a comprehensive observation, it is found that DWT technique are able to compress the image data with less time taken while Huffman technique are suitable in maintaining the quality of image but need a long time to process. Hence, hybrid of DWT with Huffman method are proposed to support each other in terms of compression, computing time and quality of image.
\end{abstract}

Key words: DWT, Huffman, Image Compression, ZigBee.

\section{INTRODUCTION}

Wireless Sensor Network (WSN) is a communication process where the data signals are transmitted through the air by using computers or devices [1]. In addition, most of users nowadays gain and exchange information via wireless network in terms of text data, graphical data, audio data [2] and etc. Image is a two dimensional (2-D) array of pixels which commonly presented as analog form which are then converted to digital form for storage purpose, processing and transmission [3] through electronic device or computer application. Consequently, rapid development of wireless network technologies are essential [4] especially in transmitting a good quality of image data.

There are several platform of WSN are used in industrial and commercial applications including ZigBee platform which are compatible for high-level communication protocol and support multi-hopping concept [5]. Although ZigBee only used a low power consumption, however, it has limitation in transmitting huge data such as image due to its small protocol which have a low data rate [6]. A high quality of image usually contain a large storage capacity and required more time to transmit an image without any loss. Thus, other than denoising an image [7], image compression plays an important role to reduce the size of image data before transmitted and improve the computing time [8] to receive the image data with lowest amount of data loss. Hence, a suitable method of compression technique are proposed to investigate the effectiveness of compressing an image to reduce the data capacity in storing and transmitting the image.

\subsection{Discrete Wavelet Transform}

Discrete Wavelet Transform (DWT) is categorized in lossy compression method which has the ability in reducing the matrix size of image [9] with low amount of resolution loss [10]. Commonly, a lossy compression usually have some loss during compression, however in DWT technique, it offers less computational complexity rather than sacrificing the quality of image [11]. The input of digitized image are decomposed into a sequence of wavelet coefficient. Besides, DWT has the advantage in computing the process in a short amount of time with less errors produced.

\subsection{Huffman Coding}

Huffman coding is an entropy-based algorithm which are capable in breaking down a long data strings into shorter ones [8]. Furthermore, image compression which uses the 
Huffman coding technique has been improved for the last three decades due to its advantage which categorized in lossless technique [12]. Huffman coding are able to rely on the analysis of the frequency of symbols in an array which can be used to compress all sorts of data efficiently. Moreover, it has the ability in choosing the representation for particular images which results in a prefix code. Thus, Huffman coding is the most efficient design in the process of storing images due to the ability in maintain the image data without any loss.

\subsection{Existing Research on Image Compression}

Nowadays, the application employs on image compression are spreads widely. Thus, a literature study on existing research are conducted to observe the performance related on this work. Table 1 shows the performance of some existing research work which used Lena image as the input image. There are some research worked on stand-alone method and hybrid method [12]. Besides, the result are listed to observe the performance in terms of compression ratio (CR), Mean Square Error (MSE), Power Signal to Noise Ratio (PSNR), Structural Similarity Index (SSIM) and time to compute the process.

A high compression ratio gives the meaning that the storage size can be save after applied the compression method. Based on the existing research result, the work done by V. Basavanthaswami and T. Somasekhar [13] able to obtain very high compression due to the stand-alone DWT method. Commonly, DWT has the ability in reducing the image size due to filtering concept in DWT method. Other than that, the computing time of image compression are also important in supporting some application that required fast compression time such as face recognition and remote sensing [14] application.

Next, MSE contribute to the amount of error which affect the PSNR value. The value of PSNR is opposite to the result obtained in MSE value. PSNR values ranged between $30 \mathrm{~dB} \sim$ $60 \mathrm{~dB}$ are acceptable values in lossy compression. In addition, SSIM value also affect the quality of reconstructed image by matching the similarity between compressed and decompressed image. The metric returns the real value ' 1 ' and 'less than 1' which donates to identical image and different image, respectively [15]. The author H. Naaz et.al [16] investigates that both stand-alone DWT and hybrid method can produce good quality of image with an acceptable amount of PSNR and SSIM value that near to ' 1 '.

Basically, a research done by H. Singh and S. Rana [12] investigates the performance in each stage of compression (DWT-DCT-Huffman). The quality of image are able to maintain after first stage with less than one seconds to compress. However, there are many error after DCT method is applied while the following stage, Huffman can compress without any data loss but took long time to complete the stage which affect the overall hybrid compressions. As conclusions, stand-alone DWT method can provide effective performance compared to hybrid method. Despite of that, both technique supports each other in certain aspect which depends on the matter of application used.

Table 1: Comparison of results based on existing research work on Lena image

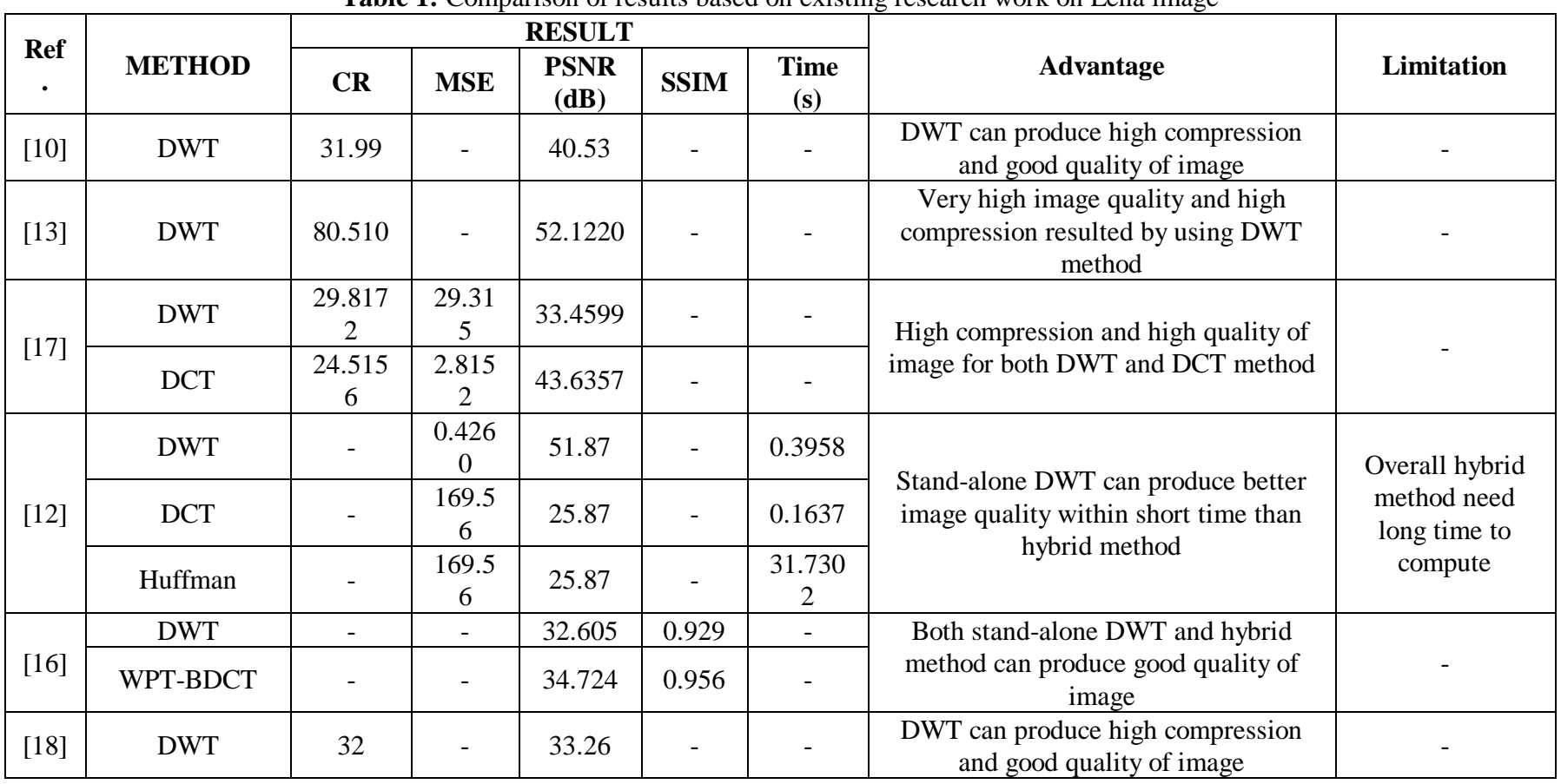




\section{METHODOLOGY}

Basically, the aim of this work are implementing a good compression method for image storing and transmission purpose wirelessly via ZigBee network. In this work, the method are divided into three section which are: (i) Stand Alone DWT Technique; (ii) Huffman Technique; and (iii) Hybrid of DWT and Huffman Coding Technique.

Figure 1 shows the flowchart of image compression process by using DWT with Huffman Coding Technique. An input image are tested by selecting a $512 \times 512 \times 3$ (width $\mathrm{x}$ length $\mathrm{x}$ layer) dimension size of coloured Lena image. The process starts by dividing the image into Red (R), Green $(\mathrm{G})$ and Blue (B) part or RGB layer. After that, Forward-DWT Compression is applied in each RGB layer, followed by Huffman Encoding process. Next, compressed image is stored or transmit wirelessly via ZigBee network. At the receiver stage, the process starts by applying Huffman Decoding process and followed by Inverse-DWT process. Then, the decompressed image are regrouped from RGB layer to become single coloured image as the original image. The output image are displayed and end the process.

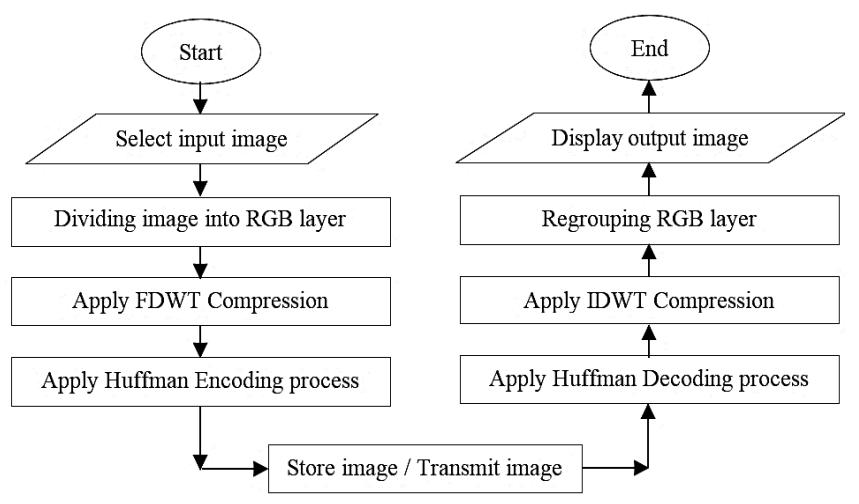

Figure 1: Flowchart of DWT with Huffman Encoding Technique

\subsection{Stand-Alone DWT Technique}

A 2-Dimensional (2D) DWT techniques applied the concept of filtering process and minimize the complexities in its architecture [8]. Besides, it transform the image between time and frequency domain which an appropriate adaptive band are selected based on signal characteristic and improve the time-frequency resolution whenever the frequency band match the signal spectrum [19].

There are many levels of DWT where it depends on the filtering stage of the transformation's occurrence can be made. Based on Figure 2, the selected image are divided into half which carry out the process of High Pass Filter (HPF) and Low Pass Filter (LPF) [8]. The process of separating image into half which based on row and column wise filter to produce smaller sub-bands are also known as level of decomposition [20]. The process of decomposition are then continued as the level increased which are visualized in Figure 3 and Figure 4.

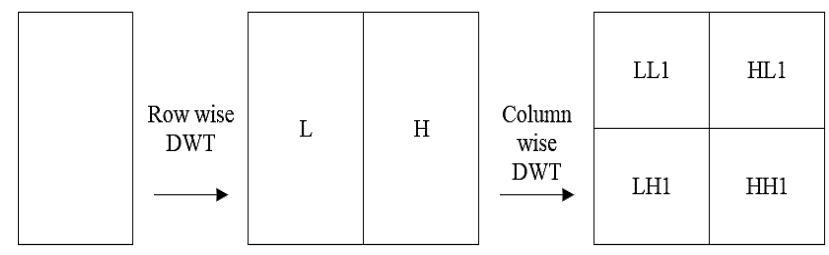

Figure 3: First Level of Decomposition

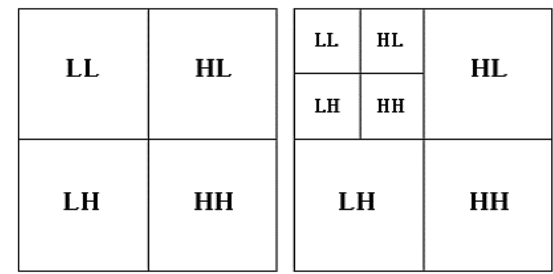

\begin{tabular}{|c|c|c|c|}
\hline IL & HL & HL & \\
\cline { 1 - 1 } LH & HH & & \multirow{2}{*}{ HL } \\
\cline { 1 - 1 } LH & HH & \\
\hline & LH & \multirow{2}{*}{ HH } \\
\hline
\end{tabular}

Figure 4: First, Second and Third Level of Decomposition

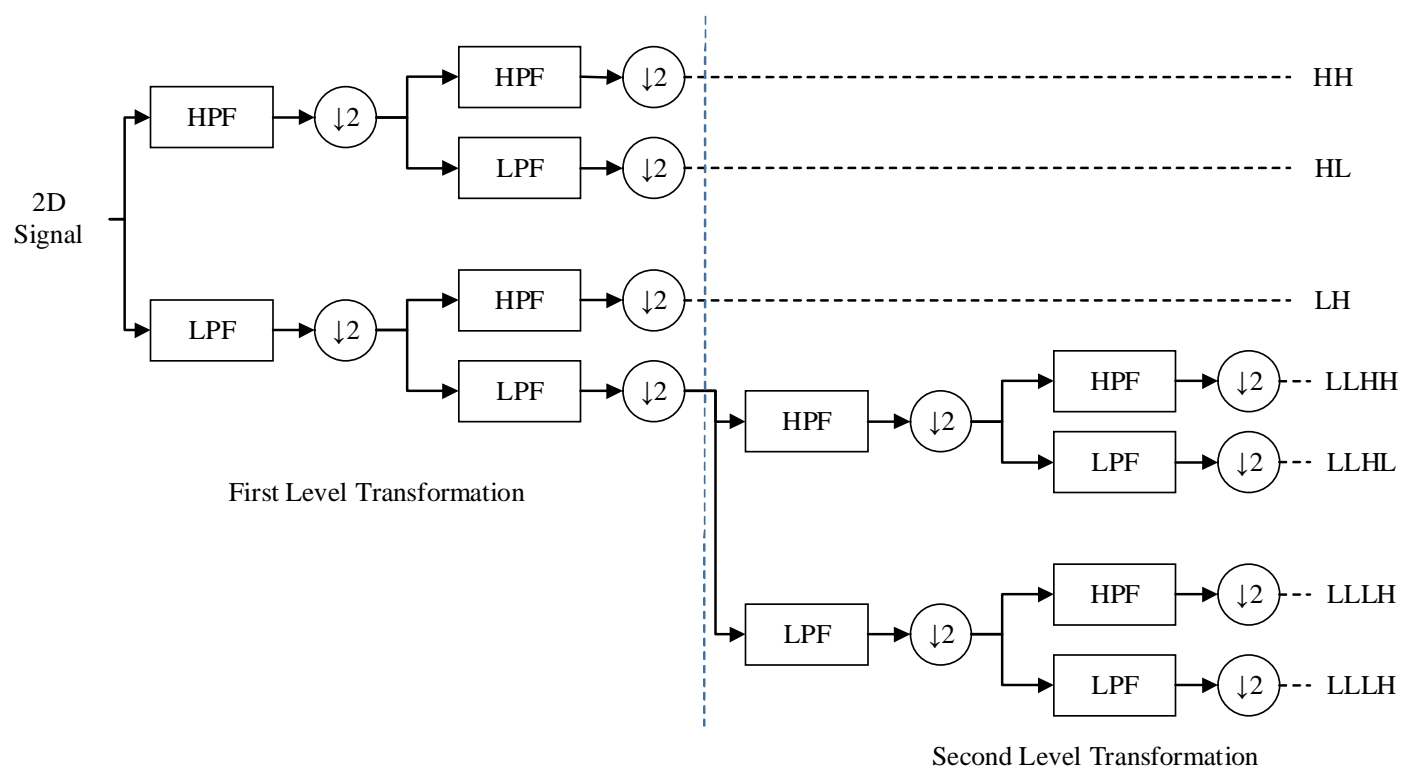


Figure 2: Filtering Stage for First and Second Transformation Level of 2D DWT Technique

\subsection{Stand-Alone Huffman Technique}

Number Huffman techniques is the process of shortening an array of data by encoding the data into another character of data by implementing a binary tree concept [21] as sample shown in Figure 5. A binary tree method gives a minimal encoded stream of data by generating an optimal tree from selected data [21]. The probabilities of occurrence are assigned to trace the path from the root to the end character or destination of the character which makes the path of $\mathrm{Y}$ is '110' shown in Figure 5. As the height of root increased, the length of encoded data also increased.

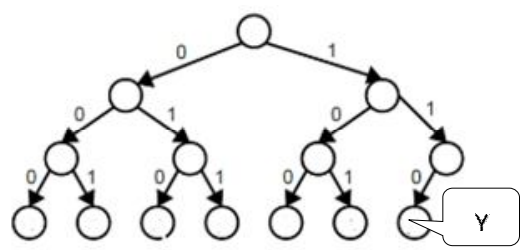

Figure 5: Binary Tree Concept

\subsection{Hybrid of DWT with Huffman Coding}

In this method, the process of DWT techniques are hybrid with Huffman Coding and tested in each level of DWT until level 6. Although DWT itself are proved in producing high quality and better performance of image compression, however Huffman Coding are certainly required in this work due to its ability in shortening a large string of data. In this work, the advantage of Huffman can be seen on the encoded data which represented as binary data.

Binary data are very essential in the application of ZigBee network because ZigBee lack in processing the image data which displaying several character that hard to programmed by ZigBee. Hence, a binary data are much easier for ZigBee network to process. Furthermore, applying a stand-alone Huffman techniques required long time to compute and large storage data to transmit wirelessly. Hence, hybrid of DWT with Huffman Coding are proposed in this work. Other than encoding the image data, it fasten the computing time and saving more data in memory and ease transmission process.

\subsection{Evaluation of Performance}

In this work, the performance of each method applied are evaluated in terms of dimension size, compression ratio (CR), Mean Square Error (MSE), Power Signal-to-Noise Ratio (PSNR), Structural Similarity Index (SSIM) and Computing Time. Most of past paper such as author H. Naaz et.al [22] and R. Kumar et.al [23] evaluated their performance of image in terms of MSE and PSNR only. However, in this paper observes performance in several terms in order to determine the best results based on method applied. The calculation of
$\mathrm{CR}$, MSE and PSNR value are expressed as the following equation:

$$
\begin{aligned}
& \text { CR }=\frac{\text { Original image size }}{\text { Compressed image size }} \\
& \text { MSE }=\frac{1}{\mathrm{mn}} \sum_{i=0}^{m-1} \sum_{j=0}^{n-1}[I(i, j)-K(i, j)]^{2} \\
& \text { for } \mathrm{m}=\text { row of image (in pixel) } \\
& \text { for } \mathrm{n}=\text { column of image (in pixel) } \\
& \text { for } K=\text { Noisy approximation } \\
& \text { for } I=\text { Noise free }(m \times n) \text { monochrome image } \\
& \text { PSNR }=10 \log \frac{\left(M A X_{I}\right)^{2}}{M S E} \\
& \text { for } M A X_{I}=\text { Maximum possible pixel value of } \\
& \text { image }
\end{aligned}
$$

\section{RESULT AND ANALYSIS}

Essentially, result of each test are evaluated by observing the performance to determine the quality of image in each method and indicate the suitable method to store and transmit via ZigBee.

\subsection{Stand-Alone DWT Technique}

In this work, image compression are observed until DWT Level 6. Table 2 shows the result of image compression by using DWT techniques from Level 1 until Level 6. The compression ratio increased as the level of DWT rise due to the dimension size of DWT image became smaller after divided into half which attribute to the concept of filtering image after applied DWT techniques. Other than that, PSNR value reduced as the level of DWT increase which due to the augmented value of error (MSE). As the dimension size of DWT smaller, the information of image data are reduced for every transformation done. This is due to the concept of decomposition which removes HPF data and took LPF data to continue making smaller sub-bands.

The reconstructed image shown in Table 4 observed that the quality of image are reduced as the level of DWT arise. In addition, the similarity index also reduced which proves that as the level of DWT increased, there are more difference between reconstructed image and original image. Next, although the computing time are longer as the levels increased, however the time taken to process the DWT method for all tested DWT levels are less than one seconds which makes DWT method is essential and gives large benefit in image compression.

\subsection{Stand-Alone Huffman Technique}

Based on Table 2, the result of applying Huffman techniques are very exciting due the classification of Huffman Coding 
lies in lossless compression where there are no error and makes infinity result in PSNR value. Furthermore, the SSIM value is 1.0000000 which proves that the original image are equal to the reconstructed image. Despite of having no data loss, the time taken to encode and decode the image data is very different to DWT with 623.333150 second (10.39 minutes) to process. Other than that, the encoded image data are very large which makes compression ratio for Huffman is higher than original image data. This is due to the process of binary tree concept which need an assumption of range of values so called as dictionary to keep the information of coordination of pixel's value in each RGB layer. Hence, Huffman required more time to process and produce a lossless output.

\subsection{Hybrid of DWT with Huffman Coding}

In The result in Table 3 shows the original dimension size of RGB layer when it has converted in array data are compared with dimension size of DWT with Huffman Coding. As the level of DWT increased, the compression ratio also increased which denotes from the DWT's concept. Next, the MSE value from Hybrid L1 decreased until Hybrid L4 and started to rise again after Hybrid L5 which can be observed in Table 4.

Table 2: Stand-Alone DWT Technique and Stand-Alone Huffman Technique

\begin{tabular}{|c|c|c|c|c|c|c|c|c|c|}
\hline \multirow{2}{*}{ Method } & \multicolumn{2}{|c|}{ Original Dimension Size } & \multicolumn{2}{|c|}{ Dimension Size of DWT } & \multirow{2}{*}{ CR } & \multirow{2}{*}{ MSE } & \multirow{2}{*}{$\begin{array}{c}\text { PSNR } \\
\text { (dB) }\end{array}$} & \multirow{2}{*}{ SSIM } & \multirow{2}{*}{$\begin{array}{c}\text { Computing } \\
\text { Time (s) }\end{array}$} \\
\hline & RGB Layer & Total Size & RGB Layer & Total Size & & & & & \\
\hline DWT L1 & $\begin{array}{l}512 \times 512 \\
512 \times 512 \\
512 \times 512\end{array}$ & 786432 & $\begin{array}{l}257 \times 257 \\
257 \times 257 \\
257 \times 257\end{array}$ & 198147 & $1: 3.9689$ & 12.25 & 37.43 & 0.9868504 & 0.090754 \\
\hline DWT L2 & $\begin{array}{l}512 \times 512 \\
512 \times 512 \\
512 \times 512\end{array}$ & 786432 & $\begin{array}{l}130 \times 130 \\
130 \times 130 \\
130 \times 130\end{array}$ & 50700 & $1: 15.5115$ & 28.86 & 33.54 & 0.9529756 & 0.084469 \\
\hline DWT L3 & $\begin{array}{l}512 \times 512 \\
512 \times 512 \\
512 \times 512\end{array}$ & 786432 & $\begin{array}{l}66 \times 66 \\
66 \times 66 \\
66 \times 66\end{array}$ & 13068 & $1: 60.1800$ & 39.17 & 32.21 & 0.9374498 & 0.080460 \\
\hline DWT L4 & $\begin{array}{l}512 \times 512 \\
512 \times 512 \\
512 \times 512\end{array}$ & 786432 & $\begin{array}{l}34 \times 34 \\
34 \times 34 \\
34 \times 34\end{array}$ & 3468 & $1: 226.7682$ & 55.97 & 30.67 & 0.9174238 & 0.071826 \\
\hline DWT L5 & $\begin{array}{l}512 \times 512 \\
512 \times 512 \\
512 \times 512\end{array}$ & 786432 & $\begin{array}{l}18 \times 18 \\
18 \times 18 \\
18 \times 18\end{array}$ & 972 & $1: 809.0864$ & 71.11 & 29.64 & 0.8991200 & 0.115606 \\
\hline DWT L6 & $\begin{array}{l}512 \times 512 \\
512 \times 512 \\
512 \times 512\end{array}$ & 786432 & $\begin{array}{l}10 \times 10 \\
10 \times 10 \\
10 \times 10\end{array}$ & 300 & $1: 2621.44$ & 85.49 & 28.84 & 0.8771708 & 0.105680 \\
\hline HUFFMAN & $\begin{array}{l}512 \times 512 \\
512 \times 512 \\
512 \times 512\end{array}$ & 786432 & $\begin{array}{l}1 \times 1910502 ; \\
1 \times 1998894 \\
1 \times 1840621\end{array}$ & 5750017 & $7.3115: 1$ & 0 & Infinity & 1.0000000 & 623.333150 \\
\hline
\end{tabular}

Table 3: Hybrid of DWT with Huffman Coding Technique

\begin{tabular}{|c|c|c|c|c|c|c|c|c|c|}
\hline \multirow[t]{2}{*}{ Method } & \multicolumn{2}{|c|}{$\begin{array}{l}\text { Dimension Size Without } \\
\text { Compression Technique }\end{array}$} & \multicolumn{2}{|c|}{$\begin{array}{l}\text { Dimension Size of DWT with } \\
\text { Huffman Technique }\end{array}$} & \multirow[t]{2}{*}{ CR } & \multirow[t]{2}{*}{ MSE } & \multirow{2}{*}{$\begin{array}{c}\text { PSNR } \\
\text { (dB) }\end{array}$} & \multirow[t]{2}{*}{ SSIM } & \multirow{2}{*}{$\begin{array}{c}\text { Computing } \\
\text { Time (s) }\end{array}$} \\
\hline & RGB Layer & Total Size & RGB Layer & Total Size & & & & & \\
\hline Hybrid L1 & $\begin{array}{l}1 \times 262144 \\
1 \times 262144 \\
1 \times 262144\end{array}$ & 786432 & $\begin{array}{l}1 \times 96904 \\
1 \times 92774 \\
1 \times 72574 \\
\end{array}$ & 262252 & $1: 2.9988$ & 74.53 & 29.60 & 0.6957650 & 12.443923 \\
\hline Hybrid L2 & $\begin{array}{l}1 \times 262144 \\
1 \times 262144 \\
1 \times 262144\end{array}$ & 786432 & $\begin{array}{l}1 \times 31539 \\
1 \times 34285 \\
1 \times 27377\end{array}$ & 93201 & $1: 8.4380$ & 90.51 & 28.60 & 0.8576353 & 6.926243 \\
\hline Hybrid L3 & $\begin{array}{l}1 \times 262144 \\
1 \times 262144 \\
1 \times 262144\end{array}$ & 786432 & $\begin{array}{c}1 \times 10912 \\
1 \times 11960 \\
1 \times 9581\end{array}$ & 32453 & $1: 24.2330$ & 56.44 & 30.62 & 0.9145333 & 3.534152 \\
\hline Hybrid L4 & $\begin{array}{l}1 \times 262144 \\
1 \times 262144 \\
1 \times 262144\end{array}$ & 786432 & $\begin{array}{c}1 \times 4015 \\
1 \times 4369 \\
1 \times 3587\end{array}$ & 11971 & $1: 65.6948$ & 55.34 & 30.72 & 0.9127064 & 1.968395 \\
\hline Hybrid L5 & $\begin{array}{l}1 \times 262144 \\
1 \times 262144 \\
1 \times 262144\end{array}$ & 786432 & $\begin{array}{l}1 \times 1603 \\
1 \times 1676 \\
1 \times 1451\end{array}$ & 4730 & $1: 166.2647$ & 72.17 & 29.57 & 0.8970646 & 1.323474 \\
\hline Hybrid L6 & $\begin{array}{l}1 \times 262144 \\
1 \times 262144 \\
1 \times 262144\end{array}$ & 786432 & $\begin{array}{l}1 \times 725 \\
1 \times 754 \\
1 \times 680\end{array}$ & 2159 & $1: 364.2575$ & 85.55 & 28.84 & 0.8769561 & 1.492217 \\
\hline
\end{tabular}

Consequently, the highest PSNR value with least error contribute in Hybrid L4. In addition, the result of SSIM value for Hybrid L3 is 0.9145333 which by about $0.18 \%$ higher than Hybrid L4 with 0.9127064. Hence, both Hybrid L3 and 
Hybrid L4 achieve a good quality of image. Other than that, time taken to compute all hybrid level became faster as the level increased. The maximum computing time is 12.44 seconds for Hybrid L1 while the minimum computing time is 1.49 seconds for Hybrid L6. This shows an improvement in computing time for hybrid method by about 50 times shorten compared to stand-alone Huffman method.

\section{CONCLUSION}

In this work, DWT method shows good compression ratio and computing time while Huffman method shows the ability in producing high image quality without loss. Despite of that, DWT method created some loss while Huffman method lack in computing time. Thus, hybrid method are necessary in producing better performance. Furthermore, it is proved that hybrid method in level 3 and level 4 obtained better performance than other hybrid level. In conclusion, hybrid of DWT with Huffman technique are essential in storing and transmitting image data via ZigBee network.

\section{ACKNOWLEDGEMENT}

The authors would like to thank Research, Innovation, Commercialization and Consultation Management (ORICC) Universiti Tun Hussein Onn Malaysia (H180 Tier 1 Fund and H303 GPPS Fund) and Registrar Office for sponsoring the research and development of this project.

\section{REFERENCES}

[1] K. Sharma and N. Dhir, "A Study of Wireless Networks: WLANs, WPANs, WMANs, and WWANs with Comparison," Int. J. Comput. Sci. Inf. Technol., vol. 5, no. 6, pp. 7810-7813, 2014.

[2] T. C. Sandanayake and A. M. Bandara, "Automated Classroom Lecture Note Generation Using Natural Language Processing and Image Processing Techniques," Int. J. Adv. Trends Comput. Sci. Eng. IJATCSE, vol. 8, no. 5, pp. 1920-1926, 2019. https://doi.org/10.30534/ijatcse/2019/16852019

[3] J. Vrindavanam, S. Chandran, and G. K. Mahanti, "A Survey of Image Compression Methods," Int. J. Comput. Appl., no. February 2012, pp. 12-17, 2012.

[4] S. A. Aliesawi, D. S. Alani, and A. M. Awad, "Secure image transmission over wireless network," Int. J. Eng. Technol., vol. 7, no. 4, p. 2758, 2018.

[5] G. Pekhteryev, Z. Sahinoglu, P. Orlik, and G. Bhatti, "Image Transmission over IEEE 802.15.4 and ZigBee Networks," ISCAS, no. May, pp. 3539-3542, 2005.

[6] W. Chantharat and C. Pirak, "Image Transmission over ZigBee Network with Transmit Diversity," Ipcsit.Com, vol. 7, pp. 139-143, 2011.

[7] B. ChinnaRao and M. Madhavi Latha, "An Image Denoising Framework based on Patch Grouping in
Complex Wavelet Domain," Int. J. Adv. Trends Comput. Sci. Eng. IJATCSE, vol. 8, no. 5, pp. 2299-2306, 2019. https://doi.org/10.30534/ijatcse/2019/68852019

[8] M. A. Hussin, F. A. Poad, and A. Joret, "A Comparative Study on Improvement of Image Compression Method using Hybrid DCT-DWT Techniques with Huffman Encoding for Wireless Sensor Network Application," Int. J. Integr. Eng., vol. 11, no. 3, pp. 149-158, 2019.

[9] A. Al-Fayadh and M. Abdulkareem, "Improved Transform Based Image Compression Methods," Appl. Math. Sci., vol. 11, no. 47, pp. 2305-2314, 2017.

[10] M. M. Rahman and M. M. Rahman, "Efficient Image Compression Technique using JPEG2000 with Adaptive Threshold," Int. J. Image Process. IJIP, vol. 9, no. 3, pp. 166-174, 2015.

[11]N. Surabhi and S. N. Unnithan, "Image Compression Techniques: A Review," IJEDR, vol. 5, no. 1, pp. 585-589, 2017.

[12]H. Singh and S. Rana, "Image Compression Hybrid using DCT , DWT , Huffman," Int. J. Sci. Eng. Res., vol. 3, no. 8, pp. 1-4, 2012.

[13] V. Basavanthaswami and T. Somasekhar, "Image Compression using SPHIT," Int. J. Innov. Res. Comput. Commun. Eng., vol. 5, no. 2, pp. 1839-1844, 2017.

[14] S. K. Dewangan, "Importance \& Applications of Digital Image Processing," vol. 7, no. 07, pp. 316-320, 2016.

[15] J. Balsa, T. Domínguez-Bolano, Ó. Fresnedo, J. A. García-Naya, and L. Castedo, "Image Transmission: Analog or Digital," in Proceedings, 2018, vol. 2, no. 18, p. 1163.

[16] V. S. Thakur, S. Gupta, and K. Thakur, "Hybrid WPT-BDCT Transform for High-Quality Image Compression," IET Image Process., vol. 11, no. 10, pp. 899-909, 2017.

[17] M. T. Banday and T. J. Shah, "A Comparative Study of Image Compression Techniques," 9th J\&K Sci. Congr. Reg. Sci. Congr., 2013.

[18]R. Naveenkumar, B. N. Jagadale, and J. S. Bhat, "Efficient Wavelet Coding Technique in DWT-DCT for Color Image Compression," J. Signal Process. Syst., vol. 4, no. 3, pp. 26-32, 2018.

[19]Z. Xizhi, "The Application of Wavelet Transform in Digital Image Processing," Int. Conf. Multimed. Inf. Technol., pp. 326-329, 2008.

https://doi.org/10.1109/MMIT.2008.134

[20] H. Zaineldin, M. A. Elhosseini, and H. A. Ali, "Image compression algorithms in wireless multimedia sensor networks: A survey," Ain Shams Engineering Journal, vol. 6, no. 2. Ain Shams University, pp. 481-490, 2015.

[21]E. Mishra and K. K. Singh, "Comparision of Various Lossless Image Compression Techniques," Int. J. Eng. Res. Appl., vol. 5, no. 6, pp. 36-39, 2015.

[22]H. Naaz, S. V Rathkanthiwar, and S. Kakde, 
"Implementation of Huffman Image Compression and Decompression Algorithm," Int. J. Eng. Res., vol. 2, no. 4, pp. 398-403, 2016.

[23] R. Kumar, S. Singh, and K.-H. Jung, "Human Visual System based Enhanced AMBTC for Color Image

Compression using Interpolation," 2019 6th Int. Conf. Signal Process. Integr. Networks, no. 1, pp. 903-907, 2019.

https://doi.org/10.1109/SPIN.2019.8711635 